\title{
Leishmania spp. Detection Using a Surface Plasmon Resonance Biosensor ${ }^{\dagger}$
}

\author{
Edlaine Ferreira ${ }^{1}$, Jefferson Lima ${ }^{1}$, Renata Pereira Alves-Balvedi ${ }^{2}$, Paulo Rogério Bonan ${ }^{1,3}$, \\ Eliton Medeiros 4, Luiz Goulart 2, Antonio Lima 5, Helmut Neff 5, Leiva Oliveira ${ }^{6}$, Lucio \\ Castellano ${ }^{1}$, Artur Araújo ${ }^{7}$ and Cleumar Moreira ${ }^{8, *}$ \\ 1 Human Immunology Research and Education Group, Universidade Federal da Paraíba, 58051-900 PB, \\ Brazil; edlainne@outlook.com (E.F.); jefferson.idalino@gmail.com (J.L.); pbonan@yahoo.com (P.R.B.); \\ luciocastellano@gmail.com (L.C.) \\ 2 Institute of Genetics and Biochemistry. Universidade Federal de Uberlândia, Uberlândia, 38408-100 MG, \\ Brazil; renata.balvedi@uftm.edu.br (R.P.A.-B.); lrgoulart@ufu.br (L.G.) \\ 3 Clinical and Social Dentistry Department, Universidade Federal da Paraíba, Joao Pessoa, 58051-900 PB, \\ Brazil \\ 4 Materials Science Engineering Department, Universidade Federal da Paraíba, Joao Pessoa, 58051-900 PB, \\ Brazil; eliton@ct.ufpb.br \\ 5 Electrical Engineering Department. Universidade Federal de Campina Grande, 58051-900 Paraiba, Brazil; \\ amnlima@dee.ufcg.edu.br (A.L.); heneff@web.de (H.N.) \\ 6 Universidade Federal Rural do Semi-Árido, Angicos, 59515-000 RN, Brazil; leivacasemiro@gmail.com \\ 7 Université de Lorraine, 54000 Nancy, France; alvesdea1@univ-lorraine.fr \\ 8 Electrical Engineering Department. Instituto Federal da Paraiba, Joao Pessoa, 58800-970 PB, Brazil \\ * Correspondence: cleumar.moreira@ifpb.edu.br; Tel.: +55-83-3612-1346 \\ + Presented at the Eurosensors 2017 Conference, Paris, France, 3-6 September 2017.
}

Published: 9 August 2017

\begin{abstract}
The detection of Leishmania spp. through a disposable surface plasmon resonance (SPR) biochip, using mono- and polychromatic sources, is presented here. A highly specific and sensitiveconstrained synthetic peptide (LC2) derived from a mimotope of Leishmania chagasi antigen was immobilized on the sensing region using a simple protocol. ELISA- and PCR-confirmed negative and positive sera of Leishmania chagasi infected patients were used. Detection of positive IgG showed a fast response with good sensitivity and specificity for the proposed method.
\end{abstract}

Keywords: Leishmania spp.; surface plasmon resonance; fast diagnosis; biosensor

\section{Introduction}

In conventional diagnosis of leishmaniasis, a study of amastigotes in smear, culture for Leishmania, histopathological examination of lesions and identification of Leishmania by polymerase chain reaction (PCR) and enzyme-linked immunosorbent assay (ELISA) should be performed. Recently label-free surface plasmon resonance method has been employed as a viable alternative for leishmaniasis diagnosis providing fast response and comparable sensitivity. Besides, another important feature of the SPR based sensors is the need for very small quantity of analyte material, such as blood samples from a patient.

\subsection{General Aspects of Leishmaniasis}

Leishmaniasis represents endemic infections caused by a protozoan of the genus Leishmania, which affects man and different animal species, wild and domestic, being transmitted by different species of mosquitoes and presenting a broad spectrum of clinical manifestations [1]. Visceral leishmaniasis is caused by the species Leishmania donovani and Leishmania infantum (Leishmania 
chagasi). Leishmania infantum is the most common species on the American continents. It is a disease that has the mosquito of the genus Lutzomyia, phlebotomine, as main vector. Visceral leishmaniasis has raised concerns of the health authorities due to the great spread of this disease in the last decades.

According to the World Health Organization are 500,000 new cases of visceral leishmaniasis for year, being a protozoonosis fatal when left untreated [2]. The forms of diagnosis are performed through clinical examination, but because of the difficulty found for safe diagnosis, laboratory methods are part of the research arsenal. The methods currently employed in visceral leishmaniasis clinical diagnoses are costly, requiring numerous steps to perform besides specialized labor, have also shown low sensitivity to detect the initial cases of the disease, generating false negatives for the disease. Therefore, it is clear the need to develop methodologies that allow a more sensitive and faster diagnosis, guaranteeing greater control of visceral leishmaniasis [3,4].

\subsection{Surface Plasmon Resonance Biosensig Technique}

According varying coupling mechanisms, two basic modes of operation normally are commonly used at the surface plasmon resonance (SPR) biosensors: Angular Interrogation Mode (AIM), where the wavelength of incident light is fixed and the angle of incidence is varied. The SPR resonance is recorded by an optical detector; Wavelength Interrogation Mode (WIM), where the angle of incidence is kept constant and incident light wavelength is varied. A spectrometer normally is employed to determine the SPR resonance.

A 4-layer SPR device is depicted in Figure 1. The optical system is responsible for the adjustments on light source to get the optical conditions (polarization, focus, position) for phenomenon excitation; a multilayer system composed by the optical materials used in the sensor manufacturing including a bio-recognition layer that interacts with the substances contained in the analyte; a microfluidic system to confine and delivery the analyte to the sensitive surface; and one Acquisition Control Processing and Display unit (ACPDU) to analyze the sensor signal.

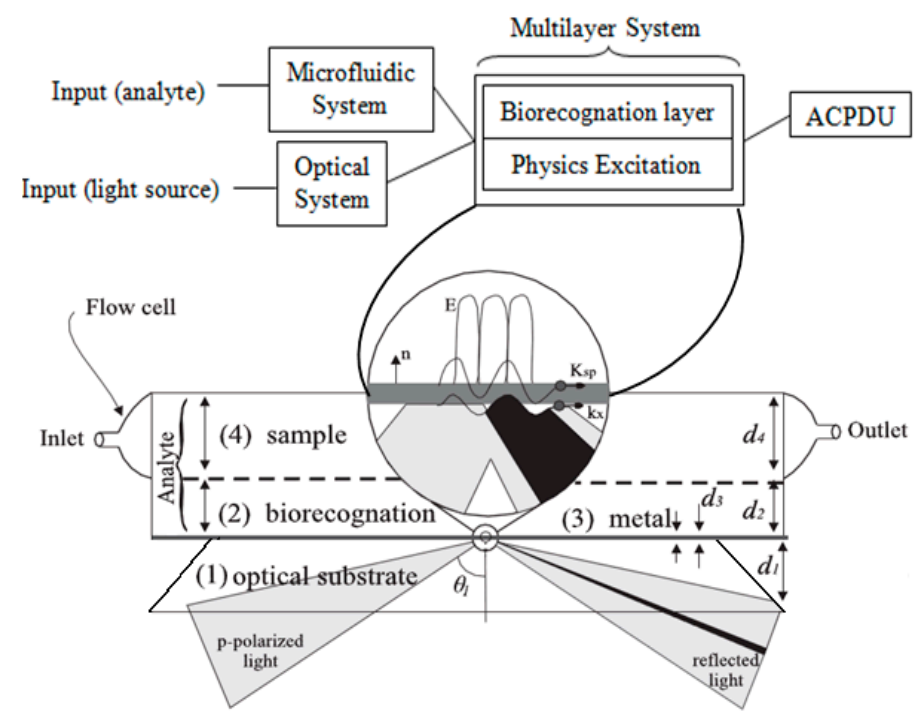

Figure 1. Block diagram of a surface plasmon resonance biosensor which comprises 4 different systems: Microfluidic, Optical, a Multilayer structure and ACPDU (Acquisition Control Processing and Display Units). Details for a typical SPR biosensor multilayer structure, whose the analyte is admitted through the flow-cell.

\section{Materials and Methods}

Two polymer SPR biochips have been employed in the experiments: one, proposed in 2004 [5] and called VIRChip. It only works at the AIM case and has diffractive optical coupling elements (DOCEs), manufactured in nanometer dimensions by using holographic techniques; another, proposed in 2009 [6], called PPBIO. It has a simple structure and can be applied for both AIM and WIM requiring relatively low design and manufacturing costs. At the experiments carried out, a 670- 
nm laser diode and a white light diode was employed at the PPBIO for AIM and WIM cases; correspondingly, a 670-nm laser diode and a CCD camera (AIM case) has been used with the VIRChip.

A highly specific and sensitive-constrained synthetic peptide (LC2) derived from a mimotope of Leishmania chagasi antigen, selected by phage display (is a selection technique in which a library of peptide or protein variants is expressed as a genetic fusion to a bacteriophage coat protein), was used as probe for IgG detection [4] were synthesized and pre-validated by the enzyme-linked immunosorbent assay (ELISA) method. Phosphate buffer saline solution ( $\mathrm{pH}$ 7.4) was used as the baseline. This peptide LC2 was modified with BSA and cysteine [7].

After biochip cleansing, -2 pg of PBS- diluted LC2 peptide was applied onto the biochips, and non-specific binding was blocked by bovine serum albumin (BSA). Diluted samples (1:1000) from healthy volunteers (negative controls) or visceral leishmaniasis infected patients (positive) were tested.

The protocol was made from the peptide LC2 modified with BSA and cysteine. Initially the gold surface was cleaned with sodium hypochlorite. $1000 \mu \mathrm{L}$ of diluted peptide was placed in PBS, after using the same amount of $\mu \mathrm{L}$ for the other steps (BSA, control of a negative human patient and control of a seropositive human patient diluted in PBS). Among the stages, one would read PBS.

\section{Results and Discussion}

Visceral leishmaniasis diagnosis has been developed by VIRChip and PPBIO biosensing systems. Sensorgrams are depicted in the Figure 2a-c, where both AIM and WIM cases are used in the PPBIO system.

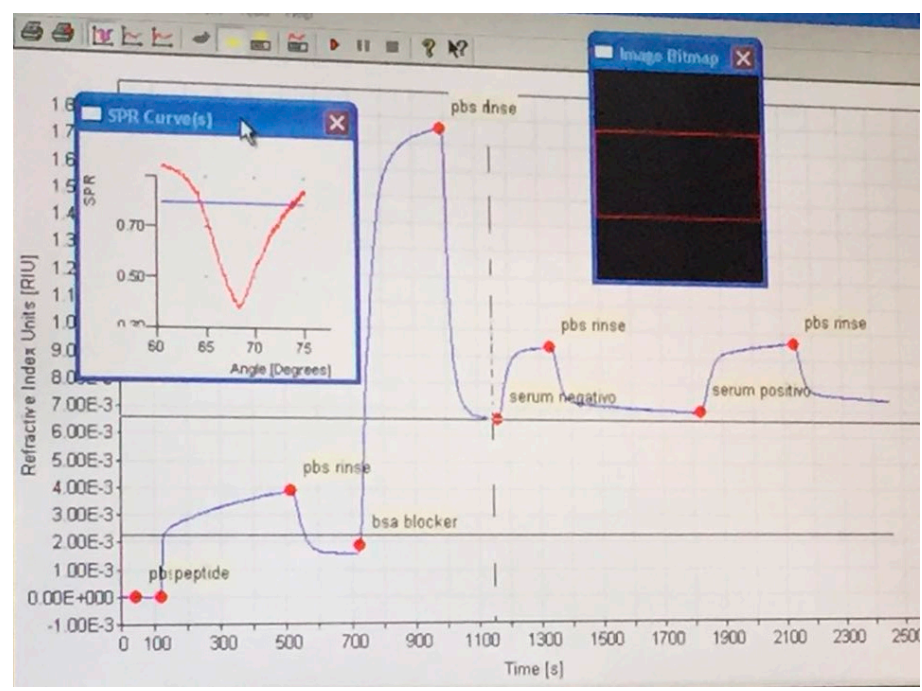

(a)

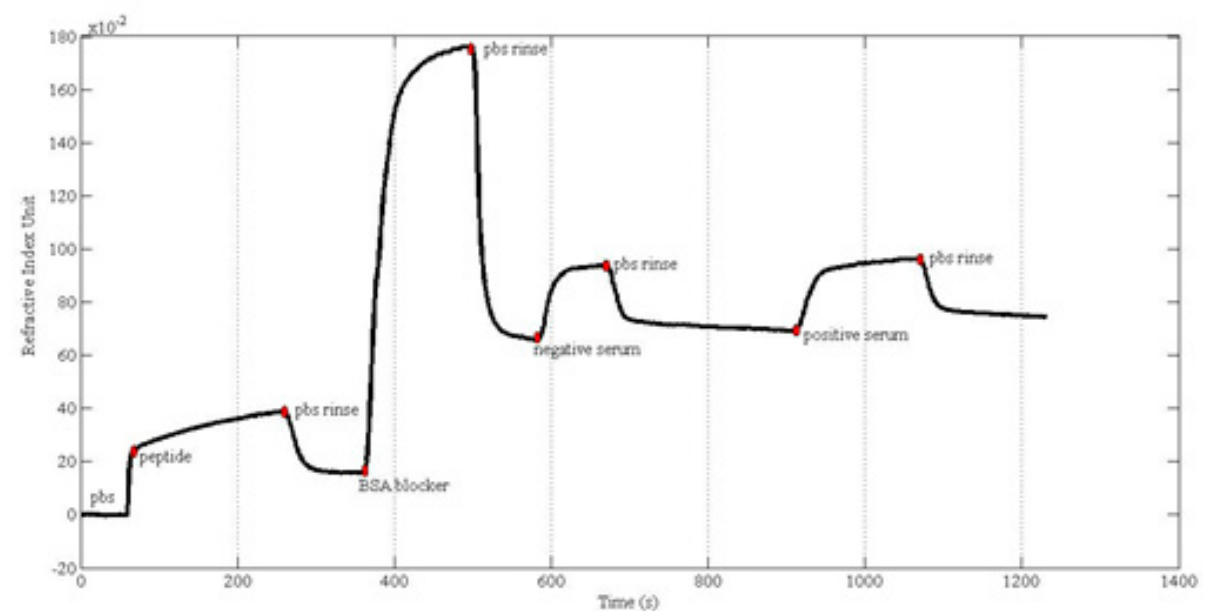

(b) 


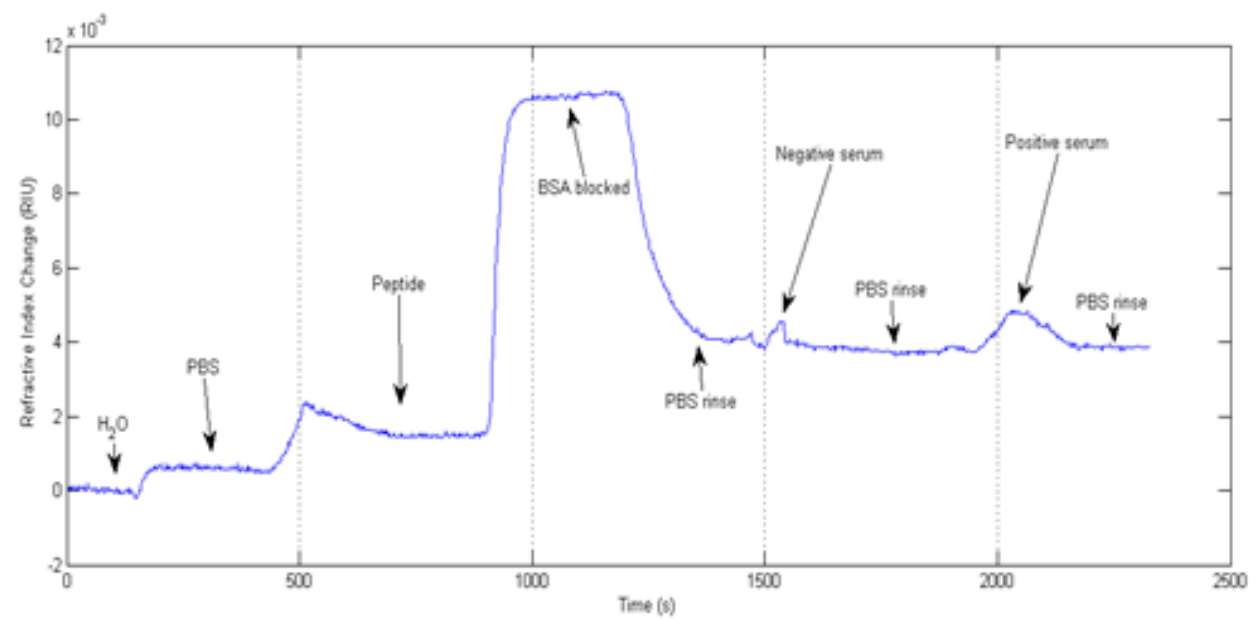

(c)

Figure 2. Sensorgrams for (a) VIRChip and PPBIO at the (b) AIM and (c) WIM cases.

Tests for positive and negative sera using the VIRChip (Figure 2a) where developed when the antigen blocked layer was adsorbed on the metallic thin layer. It took $7 \mathrm{~min}$ approximately, which lesser than conventional methods, like ELISA. The fast and correct, response, almost in real time, was observed for both serum. A sensitivity of $3300^{\circ} / \mathrm{RIU}$ was achieved in these experiments.

Sensorgrams for AIM (Figure 2b) and WIM (Figure 2c) were also developed for negative and positive sera. Higher refractive indexes for positive samples were obtained when compared to negative controls. A sensitivity of $3800 \mathrm{~nm} / \mathrm{RIU}$ has been achieved for the polychromatic source-based biochip.

\section{Conclusions}

Highly specific interactions between the LC2 peptide and circulating anti-Leishmania antibodies in sera of infected patients were detected. The current proof-of-concept for diagnosis of leishmaniasis using disposable biochips suggests this biosensor as a potential diagnostic tool that may be applied in other infectious and chronic diseases.

The efficacy of the protocol was observed in real-time confirmation of the negative and positive patients in relation to the specific peptide, showing that the immunosensor can be a highly rapid, sensitive, specific and cheaper diagnosis in relation to the other diagnoses.

Acknowledgments: The authors would like to thank $\mathrm{CNPq}$ for the financial support and research grants.

Conflicts of Interest: The authors declare no conflict of interest.

\section{References}

1. Ashford, R.W. The leishmaniases as emerging and reemerging zoonoses. Int. J. Parasitol. 2000, 30, 1269-1281.

2. Alvar, J.; Vélez, I.D.; Bern, C.; Herrero, M.; Desjeux, P.; Cano, J.; Jannin, J.; Boer, M. Leishmaniasis Worldwide and Global Estimates of Its Incidence. PLoS ONE 2012, 7, e35671.

3. Castellano, L.R.C. Resposta imune anti-Leishmania e mecanismos de evasão. VITAE Academia Biomédica Digital. Número 25 Octubre-Diciembre 2005.

4. Goulart, L.R.; Vieira, C.U.; Freschi, A.P.P.; Capparelli, F.E.; Fujimura, P.T.; Almeida, J.F.; Ferreira, L.F.; Goulart, I.M.B.; Brito-Madurro, A.G.; Madurro, J.M. Biomarkers for Serum Diagnosis of Infectious Diseases

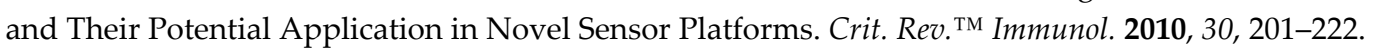

5. Thirstrup, C.; Zong, W.; Borre, M.; Neff, H.; Pedersen, H.C.; Holzhueter, G. Diffractive optical coupling element for surface plasmon resonance sensors. Sens. Actuators B 2004, 100, 298-308.

6. Moreira, C.d.S.; Oliveira, L.C.; Fischer, R.; Medeiros, E.S.; Lima, A.M.N.; Neff, H. Polymer-based surface plasmon resonance biochip: construction and experimental aspects. Res. Biomed. Eng. 2016, 32, 92-103. 
7. Goulart; R, L.; Cardoso, R.; Almeida, J.F.; Messias, F.F.; Capparelli, F.E.; Madurro, A.G.B.; Madurro, A.G.B.; Prudêncio, C.R.; de Souza, G.R.L.; Freschi, A.P.P. Peptídeos Recombinantes e Motivos Proteicos Miméticos a Antígenos de Leishmania e Suas Aplicações. Patent Application No. PI 0903089-1 A2, 12 August 2008. article distributed under the terms and conditions of the Creative Commons Attribution (CC BY) license (http://creativecommons.org/licenses/by/4.0/). 